\title{
Coronaviruses and COVID-19 - Complications and Lessons Learned for the Future
}

\author{
Muhammad Bilal ${ }^{1 *}$ (D), Muhammad Imran Khan² ${ }^{(D)}$, Muhammad Shahzad \\ Nazir $^{3}$ (D) Ishtiaq Ahmed ${ }^{4}$ iD and Hafiz M.N. Iqbal ${ }^{5 *}$ (D)
}

\begin{abstract}
${ }^{1}$ School of Life Science and Food Engineering, Huaiyin Institute of Technology, Huaian 223003, China. ${ }^{2}$ Hefei National Lab for Physical Sciences at the Microscale and the Centers for Biomedical Engineering, University of Science and Technology of China, Hefei, Anhui 230027, China. ${ }^{3}$ Faculty of Automation, Huaiyin Institute of Technology, Huaian 223003, China. ${ }^{4}$ School of Medical Science, Menzies Health Institute Queensland, Griffith University (Gold Coast campus), Parklands Drive, Southport, QLD 4222, Australia. ${ }^{5}$ Tecnologico de Monterrey, School of Engineering and Sciences, Campus Monterrey, Ave. Eugenio Garza Sada 2501, Monterrey, N.L., CP 64849, Mexico.
\end{abstract}

\begin{abstract}
Despite the earlier two Coronavirus outbreaks, this appearance of the third Coronavirus outbreak has brought several complications and now emerged as a human-health related pandemic issue. The wide geographical spread and transmissibility of newly emerged novel-Coronavirus (2019-nCoV) and severity of coronavirus disease 2019 (COVID-19) raised serious concerns about the future trajectory of the 2019nCoV/COVID-19 outbreak. Herein, we have briefly covered some important aspects of the 2019-nCoV/ COVID-19 outbreak to highlight the need to learn lessons for a safer future. First, an epidemiological comparison of respiratory viral infections with particular reference to 2019-nCoV is given with relevant examples. Following that, considering the earlier two Coronavirus outbreaks, the emergence of another new 2019-nCoV is given. Finally, the lesson learned, so far, and key concerns for pandemic impact assessment at large and COVID-19, in particular, are discussed for future considerations.
\end{abstract}

Keywords: Coronavirus, COVID-19, Zoonotic infections, Common cold, Pandemic Impact Assessment

*Correspondence: bilaluaf@hotmail.com; hafiz.iqbal@tec.mx; +52 (81) 83582000 ext. 5679 (HMN Iqbal).

(Received: March 25, 2020; accepted: March 29 2020)

Citation: Bilal M, Khan MI, Nazir MS, Ahmed I, Iqbal HMN. Coronaviruses and COVID-19 - Complications and Lessons Learned for the Future. J Pure Appl Microbiol. 2020;14(suppl 1):725-731. doi: 10.22207/JPAM.14.SPL1.09

(C) The Author(s) 2020. Open Access. This article is distributed under the terms of the Creative Commons Attribution 4.0 International License which permits unrestricted use, sharing, distribution, and reproduction in any medium, provided you give appropriate credit to the original author(s) and the source, provide a link to the Creative Commons license, and indicate if changes were made. 


\section{INTRODUCTION}

Coronaviruses and respiratory syndromes beyond the common cold

The emergence of human-health related COVID-19 pandemic has become extremely severe and deadly. According to one estimate, the pandemic of influenza caused the death of more than fifty million peoples around the world (Morens et al., 2020; Short et al., 2018). Coronaviruses have been considered as common pathogens for the common cold. But, the $21^{\text {st }}$ century has seen two episodes of global epidemics termed as middle east respiratory syndromes (MERS-CoV) and severe acute respiratory syndromes (SARS-CoV) resulting in hundreds of deaths. Figure 1 illustrates a schematic representation of the epidemiological comparison of respiratory viral infections. Until the start of the $21^{\text {st }}$ century, scientists paid little attention to coronaviruses. Due to the 2002 outbreak of SARS characterized by atypical pneumonia caused by a coronavirus, they became an essential concern of the scientific community. A large number of patients (almost $1 / 3^{\text {rd }}$ ) with the onset of fever, dyspnea, cough, and watery diarrhea needed mechanical ventilation, and the mortality rate was as high as $10 \%$. The second episode of coronaviruses infection outbreak was observed in 2012 and known as MARS. Symptoms of MARS are similar to that of SARS, but $50-89 \%$ of patients of MARS essentially needed mechanical ventilation, and the death rate was $36 \%$ (Paules et al., 2020).

The third pandemic, caused by COVID-19, also belong to coronaviruses family, which caused severe acute respiratory syndrome, alarmed global public health authorities to take some timely measures to control the pandemic switch (Iqbal et al., 2020; Morens et al., 2020). Seventeen years later, similar cases or even more severe situation appeared in the form of the pandemic of COVID-19 prevalence. Several patients, especially patients, admitted to intensive care units, also presented with breathing difficulty and neurologic disturbances like nausea, headache, and vomiting (Li et al., 2020). According to the world health organization report, almost 334981 confirmed patients, and 14652 deaths have been reported in 190 countries, territories or areas (World Health Organization, 2020), and new cases and deaths are keep growing on a daily basis. Although at the current statistics, the mortality rate of COVID-19 is considered lower than MARS and SARS episodes (Paules et al., 2020), however, the complete outcome of this pandemic is unclear yet.

\begin{tabular}{|c|c|c|c|c|}
\hline \multicolumn{5}{|c|}{ Epidemiological Comparison of Respiratory Viral Infections } \\
\hline Disease & Flu (Common cold) & COVID-19 & SARS & MERS \\
\hline \multicolumn{5}{|l|}{$\begin{array}{r}\text { Disease Causing } \\
\text { Pathogen }\end{array}$} \\
\hline $\begin{array}{r}\mathbf{R}_{0} \\
\text { Basie Reproductive Number }\end{array}$ & 1.3 & $2.0-2.5 *$ & 3 & $0.3 \cdot 0.8$ \\
\hline $\begin{array}{l}\text { CFR } \\
\text { Case Fatality Rate }\end{array}$ & $0.05 \cdot 0.1 \%$ & $\sim 3.4 \% *$ & $9.6-11 \%$ & $34.4 \%$ \\
\hline Incubation Time & 1.4 days & $4-14$ days * & $2 \cdot 7$ days & 6 days \\
\hline Hospitalization Rate & $2 \%$ & $\sim 19 \% *$ & Most cases & Most cases \\
\hline Community Attack Rate & $10-20 \%$ & $30.40 \%$ * & $10 \cdot 60 \%$ & $4 \cdot 13 \%$ \\
\hline Annual Infected (global) & $\sim 1$ billion & N/A (ongoing) & 8098 (in 2003) & 420 \\
\hline Annual Infected (US) & 10.45 million & N/A (ongoing) & 8 (in 2003) & 2 (in 2014) \\
\hline Annual Deaths (US) & $10.000 \cdot 61.000$ & N/A (ongoing) & None (since 2003) & None (since 2014) \\
\hline
\end{tabular}

* covid-19 data as of March 2020.

Fig. 1. A schematic representation of an epidemiological comparison of respiratory viral infections. The Figure was created with the "BioRender.com" template and exported under the terms of premium subscription. 
Zoonotic infections, as either pandemic, epidemic, and endemic, have a damaging effect and impose a significant threat to human life, public security, health, and national economic growth (Allen et al., 2017). Besides, the emergence of such diseases may point out our lack of understanding of natural phenomena. Now the world is turning into a global village of 7.6 billion huge population with new and advanced technologies. This significant change has resulted in a new ecosystem for host-pathogen relationship and interaction. The rapid evolution of the RNA viral genome draws another sketch of the repeated emergence of viral epidemics with more severity (Morens et al., 2020). The rise of three episodes of coronavirus originating from wildlife in a period of 20 years raises a question of human behaviors and the environment of transforming the living ecosystem. It is also alarming and challenging the scientific community to prepare for a more complicated situation to keep human beings at the safe end.

The emergence of another Coronavirus

It is important to obtain as many clinical isolates as possible that are unbound to time and geography to assess the degree of the mutation of the virus. COVID-19 may behave more like SARS-CoV and will continue to adapt to the human hosts, thereby improving binding to hACE (Huang \& Herrmann, 2020). Collecting patient samples during autopsy can help clarify the pathogenesis of infection and rationalize treatment interventions. It will also help to verify the results of diseases in laboratory animals. The second key issue is to determine the origin of the zoonotic disease of the virus. Because bats are very similar to bat coronavirus, bats may be the primary host for the virus. SARS-CoV is transmitted to humans by exotic animals in the wet market, while MERS$\mathrm{CoV}$ is transmitted from humans to camels (Hui et al. 2020). It is essential to understand whether COVID-19 is transmitted directly through bats or intermediate hosts and help define how zoonotic transmission is transmitted.

As reported by Zhu et al. COVID-19 has been identified and characterized (Xu et al. 2020). The viral genome has been sequenced, and these results and other reports show that it has $75 \%$ to $80 \%$ homology to SARS-CoV and is more closely related to various bat coronaviruses.
It can support the growth of SARS-CoV and MERS-CoV, but it should be noted that COVID-19, unlike SARS-CoV or MERS-CoV, grows better on epithelial cells of the primary human respiratory tract than on standard tissue culture cells (Zhou et al. 2020). Identification of the virus will enable the development of reagents that respond to the key unknowns of this new coronavirus infection and will guide the development of antiviral therapies. First, understanding the genome sequence can help develop reverse transcriptase and polymerase sensitive chain reaction assays for rapid virus detection. Second, the development of serological test methods will assess the prevalence of potential human zoonotic virus infections in humid markets and other environments. Because of the wet product market across China, the abovementioned methods can also be used to assess whether human infections are more common than initially thought. Third, mastery of the virus will stimulate efforts to develop antiviral therapies and vaccines.

The government's response to COVID-19 is far from perfect but contrasts sharply with the SARS epidemic. The quick launch of the COVID-2019 sequence enables the research community to participate quickly, provide analysis, and develop diagnostic tests. The Chinese Centers for Disease Control and Prevention and the Wuhan Municipal Health Commission have regularly released updated information on the number of confirmed cases and patient status so that health officials can monitor the situation in real-time. Researchers from around the world have logged on social media to compare the latest sequence information and highlight critical unknowns about the epidemic (Gralinski \& Menachery, 2020). The availability of data updates through public health officials, around the world, offers significant potential to timely response to the ongoing COVID-19 situation at local, regional, national and international levels. This connectivity has promoted awareness, new collaborations, and rapid response in the global research community. Although there are many unknowns for COVID-19, the world is determined and ready to fight the latest coronaviruses strains.

Lesson learned - Share Weal and Woe

Given the challenges faced by government authorities and suspects/infected persons/the 
healthy public, much is known about this infection. Above all, the extent of human-to-human transmission and the extent of clinical disease must be determined. SARS-CoV and MERS-CoV are widely spread through cloud events. The COVID-19 broadcast includes cloud events, but their relative importance is unknown. SARS-CoV and MERS-CoV infect lung epithelial cells more than upper respiratory cells (Camp \& Nowotny, 2020). Therefore, transmission mainly comes from patients with a recognized disease, not from patients with mild and non-specific signs. 2019nCoV appears to use the same cellular receptor as SARS-CoV (human angiotensin-converting enzyme [hACE2]), so it is not expected to spread without signs of lower respiratory tract disease. SARS-CoV was mutated during the epidemic of 2002-2004, which can better bind to its cellular receptors and optimize replication in human cells, thereby increasing toxicity. It often causes mutation and recombination events. However, no significant mutations have occurred since MERS-CoV was discovered in 2012 to increase human infectivity (Weston \& Frieman, 2020).

The COVID-19 was originally discovered in Wuhan, China, in people exposed to seafood or wet markets. The rapid response of China's clinical, scientific, and public health agencies has increased knowledge of clinical diseases and a preliminary understanding of the epidemiology of infections. Early reports indicate that personto-person transmission is limited or non-existent, but we now know that such transmission has occurred, although to what extent it is unclear. Like outbreaks caused by two other pathogenic human respiratory coronaviruses (severe acute respiratory coronavirus syndrome [SARS-CoV] and Middle Eastern respiratory coronavirus syndrome [MERSCoV]), nCoV 2019-nCoV (COVID-19) induced respiratory diseases, which are also severe. By January 24, 2020, over 800 cases, with a mortality rate of $3 \%$ had been reported (promedmail.org). The news of COVID-19 is widely known on the Internet. For years, websites such as FluTrackers. com, ProMED (promedmail.org) have helped collect disease information worldwide and disseminate it more widely to stakeholders. In 2012, MERS-CoV first referred to ProMED Mail as a "new coronavirus" and then attracted Twitter among scientific journalists, virologists, and public health experts (He et al. 2020; Giwa \& Desai, 2020). Eight years later, a more interconnected network quickly canceled the statement from the Wuhan Municipal Health Commission and speculated on possible causes. At the beginning of the epidemic, it is challenging to distinguish rumors containing truth from baseless fear. Language barriers and confidential information compound this. However, in this case, speculations on new coronaviruses are supported by carefully worded statements that specifically exclude individual virus families (influenza, adenoviruses), but exclude SARS-CoV and MERS-CoV (Goh et al. 2020). In addition to the history of the SARS epidemic, many people worry that the truth is hidden. When infected were finally identified as CoV, the world was worried and relieved that the outbreak would not continue to hide. Perhaps this means that the lessons learned from the SARS epidemic have indeed been learned. Key Concerns for Pandemic Impact Assessment

It is a common observation of natural events that epidemics and pandemics complete their course. Witnessed by observations, epidemics and pandemics follow four phases. The first phase is termed as lag phase, in which pathogen infects community and becomes stable in the affected area, but maximum numbers of peoples are asymptomatic. Time to clinical symptoms to appear may vary from case to case. The following phase appears as a rapid prevalence of infection to the whole community, and there is a rapid increase in newly reported cases during this phase. This phase is followed by the stationary phase are; in which a large number of patients are reported on a daily basis, but the number of cases may be observed almost at the same rate. Then the last and the final phase starts showing the decline in the number of patients. In the last phase, existing patients undergo recovery very rapidly, and the recovery graph improves quickly. The graph of newly diagnosed cases shows a decline (Gonzalez et al. 2018; Ray \& Reich, 2018). The duration of all these phases depends upon the response public to adopt the preventive measures and response of authorities to implement the policies in time. Despite government policies, the public response remains the primary factor in determining the duration of different phases. Usually, the infection becomes more prevalent and takes more time to eliminate when peoples do not take adequate 
preventive measures in the first response. Several other questions that include various risk factors associated with disease transmission, the pattern of disease prevalence, preventive measures, duration of epidemics and pandemics and retention of the pathogen as a common pathogen in community and re-emergence as epidemic or pandemic agents in the community or global world or studied in epidemiology and postulated on current or previous observations of disease characteristics (Liu, 2018).

The initial consideration in people was about the high death rate of COVID-19 infected peoples, and the rate of prevalence was highly contagious pathogen caused fear in public. That fear affected peoples positively and accepted infection control instructions having a positive on infection control. However, later assessment resulted that COVID-19 is less pathogenic than the MERS-CoV. COVID-19 emergence has raised some important questions regarding disease pyramid shape, the percentage of infected patients developing the disease, and the rate of peoples seeking health care during this episode of coronavirus families (Munster et al., 2020). China has done hard work to control the prevalence of COVID-19 infection successfully and has answered the questions up to certain limits. Among a total of 81457 confirmed cases, 3261 (4\%) patients died and $71883(88.25 \%)$ recovered. Remaining patients are under treatment, either standard isolation or in serious condition at the hospital. In terms of new cases, COVID-19 peaked around the mid of February, which is more than two months after the first reported case and three weeks after the declaration of COVID-19 emergency (COVID-19 geographical distribution, 2020; Zhou et al., 2020). This is to keep in mind that the first case of COVID-19 was confirmed on 12 December 2019 (Zhou et al., 2020).

Second, an essential factor that determines the duration of the epidemic or pandemic is the incubation period of infection. Because, when the pathogen remains asymptomatic for longer in the body, it provides a safe opportunity for the pathogen to transmit infection at broad scales, and it becomes a more important question when the pathogen is highly contagious COVID-19 (Bilal et al., 2020; Munster et al., 2020). A similar transmission pattern was observed from an asymptomatic Chinese business woman to her German business partner, including three other patients working in a company in a meeting during January, either in direct contact with Chinese or with German during the symptomatic incubation period (Rothe et al., 2020).

Another important question that arises about disease control is that "is the single spill-over or sneeze carries enough pathogens to transmit disease?" This question is concerned about the contagiousness of COVID-19. In a report published in China, aiming to aware of the public of threat and insist them wear a mask, they reported the transmission of COVID-19 to two patients who have just 15 seconds and 53 seconds unprotected contact with the infected patient, and they have not confirmed that either transmission was induced by sneezing or simple breathing (Ji Yuqiao, 2020).

Instead of several difficulties and challenges like the emergence of new pathogens and was the first time to report and carry on research to investigate and uncover several aspects of pathogen and its pathogenicity under the situation of the high prevalence of highly contagious infectious agent. China has provided an excellent opportunity to learn a lesson by taking an active initiative to implement policies in its early response to combat and overcome this threat. However, the convergence of COVID-19 as pandemic has flagged another warning to several other countries, especially the countries with limited resources and economies. Further questions that have not been answered and need to be investigated include; the re-emergence of COVID-19 as either endemic or pandemic agent in affected areas or population. Mutation in the COVID-19 genome resulting in another pandemic or epidemic in the future. The lifelong protection of patients infected once with COVID-19. The proportion of patients recovered before clinical symptoms. COVID-19 is a risk factor for the other complications like the hepatitis viruses for diabetes and cancer (Almani et al. 2012; Tu et al. 2017). Association of economy, pre-existing diseases such as heart diseases, diabetes, and age on outcome of infected patients. Because COVID-19 infection is associated with a breathing problem, in such a case, the outcome in patients with pre-existing respiratory issues or smokers ( $\mathrm{Li}$ 
et al., 2020). These questions, along with other unanswered questions, would be answered in the future by further ongoing researches by different researchers.

\section{ACKNOWLEDGMENTS}

All listed author(s) are thankful to their representative universities/institutes for providing the related support to compile this work.

\section{CONFLICT OF INTEREST}

The listed author(s) declare no conflict of interest in any capacity, including competing or financial.

\section{AUTHORS' CONTRIBUTION}

All listed author(s) have made a substantial, direct and intellectual contribution to the work, and approved it for publication.

\section{FUNDING}

None.

\section{ETHICS STATEMENT}

This article does not contain any studies with human participants or animals performed by any of the authors.

\section{AVAILABILITY OF DATA}

Not applicable.

\section{REFERENCES}

1.

Allen T, Murray KA, Zambrana-Torrelio C, et al. Global hotspots and correlates of emerging zoonotic diseases. Nature Communic. 2017;8(1):1124. https://doi. org/10.1038/s41467-017-00923-8

2. Almani MIK, Kazmi A, Mansoor R. Prevalence of Diabetes Mellitus in Patients of Chronic Hepatitis C. J Islamab Med Dental College. 2012;1(4):183-186.

3. Bilal M, Nazir MS, Parra-Saldivar R, Iqbal HM. 2019-nCoV/COVID-19 - Approaches to Viral Vaccine Development and Preventive Measures. J Pure Appl Microbiol. 2020;14(1):25-29. https://doi. org/10.22207/JPAM.14.1.05

4. Camp JV, Nowotny N. The knowns and unknowns of West Nile virus in Europe: what did we learn from the 2018 outbreak?. Expert Review of Anti-infective Therapy. 2020;18(2):45-154. https://doi.org/10.108 $0 / 14787210.2020 .1713751$

5. COVID-19 geographical distribution. Available online at: https://jobtube.cn/global/ [Last accessed: March 24, 2020].

6. Giwa A, Desai A. Novel coronavirus COVID-19: an overview for emergency clinicians. Emergency
Medicine Practice. 2020;22(2 Suppl 2):1-21.

7. Goh KJ, Kalimuddin S, Chan KS. Rapid Progression to Acute Respiratory Distress Syndrome: Review of Current Understanding of Critical Illness from COVID-19 Infection. Ann Acad Med Singapore. 2020;49(1):1-9.

8. Gonzalez J-P, Souris M, Valdivia-Granda W. Global Spread of Hemorrhagic Fever Viruses: Predicting Pandemics. In M. S. Salvato (Ed.), Hemorrhagic Fever Viruses. 2018;1604(3-31), Springer New York. https:// doi.org/10.1007/978-1-4939-6981-4_1

9. Gralinski LE, Menachery VD. Return of the Coronavirus: 2019-nCoV. Viruses. 2020;12(2):135. https://doi. org $/ 10.3390 / \mathrm{v} 12020135$

10. He F, Deng Y, Li W. Coronavirus Disease 2019 (COVID-19): What we know?. Journal of Medical Virology. 2020. https://doi.org/10.1002/jmv.25766

11. Huang $Q$, Herrmann A. Fast assessment of human receptor-binding capability of 2019 novel coronavirus (2019-nCoV). bioRxiv. Posted February 03, 2020. https://doi.org/10.1101/2020.02.01.930537.

12. Hui DS, I Azhar E, Madani TA, et al. The continuing 2019-nCoV epidemic threat of novel coronaviruses to global health-The latest 2019 novel coronavirus outbreak in Wuhan, China. International Journal of Infectious Diseases. 2020;91:264-266. https://doi. org/10.1016/j.ijid.2020.01.009

13. Iqbal HM, Romero-Castillo KD, Bilal M, ParraSaldivar R. The Emergence of Novel-Coronavirus and its Replication Cycle-An Overview. J Pure Appl Microbiol. 2020;14(1):13-16. https://doi. org/10.22207/JPAM.14.1.03

14. Ji Yuqiao. Transmission of coronavirus within seconds highlights need of wearing masks. Available online at: [Http://www.globaltimes.cn/content/1178740.shtml]. [Last accessed: March 24, 2020]

15. Li Y, Bai W, Hashikawa T. The neuroinvasive potential of SARS-CoV2 may be at least partially responsible for the respiratory failure of COVID-19 patients. Journal of Medical Virology. 2020. https://doi.org/10.1002/ jmv. 25728

16. Liu, L. Introduction. In Heart Failure: Epidemiology and Research Methods. 2018; 1-12. Elsevier. https://doi. org/10.1016/B978-0-323-48558-6.00001-3

17. Morens DM, Daszak P, Taubenberger JK. Escaping Pandora's Box - Another Novel Coronavirus. New England Journal of Medicine. 2020; https://doi. org/10.1056/NEJMp2002106

18. Munster VJ, Koopmans M, van Doremalen N, van Riel $D$, de Wit E. A Novel Coronavirus Emerging in China Key Questions for Impact Assessment. New England Journal of Medicine. 2020;382(8):692-694. https:// doi.org/10.1056/NEJMp2000929

19. Paules $\mathrm{Cl}$, Marston HD, Fauci AS. Coronavirus Infections-More Than Just the Common Cold. JAMA. 2020;323(8):707. https://doi.org/10.1001/ jama.2020.0757

20. promedmail.org [Last accessed: March 24, 2020].

21. Ray EL, Reich NG. Prediction of infectious disease epidemics via weighted density ensembles. PLOS Computational Biology. 2018;14(2):e1005910. https:// doi.org/10.1371/journal.pcbi.1005910 
22. Rothe $C$, Schunk $M$, Sothmann $P$, et al. Transmission of 2019-nCoV Infection from an Asymptomatic Contact in Germany. N Engl J Med. 2020;382(10):970-971. https://doi.org/10.1056/NEJMc2001468

23. Short KR, Kedzierska K, van de Sandt CE. Back to the Future: Lessons Learned From the 1918 Influenza Pandemic. Front Cell Infect Microbiol. 2018;8:343. https://doi.org/10.3389/fcimb.2018.00343

24. Tu T, Buhler S, Bartenschlager R. Chronic viral hepatitis and its association with liver cancer. Biological Chemistry. 2017;398(8):817-837. https:// doi.org/10.1515/hsz-2017-0118

25. Weston S, Frieman MB. COVID-19: Knowns, Unknowns, and Questions. mSphere. 2020;5:e00203-20. https://
doi.org/10.1128/mSphere.00203-20

26. World Health Organization. Coronavirus disease (COVID-19) Pandemic (Update). Available online at: https://www.who.int/emergencies/diseases/novelcoronavirus-2019. [Last accessed: March 24, 2020]

27. $\mathrm{Xu} \mathrm{Z}$, Shi L, Wang $Y$, et al. Pathological findings of COVID-19 associated with acute respiratory distress syndrome. The Lancet Respiratory Medicine. 2020. https://doi.org/10.1016/S2213-2600(20)30076-X

28. Zhou P, Yang XL, Wang XG, et al. A pneumonia outbreak associated with a new coronavirus of probable bat origin. Nature. 2020;579(7798):270-273. https://doi. org/10.1038/s41586-020-2012-7 